\title{
ETUDES SUR LE TRANSIT DIGESTIF CHEZ LE PORC
}

\author{
A. RERAT et J. LOUGNON \\ Station de Recherches sur l'Elevage des Porcs, \\ Centre national de Recherches zootechniques, fouy-en-fosas (Seine-et-Oise)
}

Ces études, actuellement à leur début, visent à préciser s'il existe une relation entre la vitesse d'apparition des protéines dans l'intestin grêle après le repas et les variations d'aminoacidémie du sang porte, ces dernières étant mesurées en collaboration avec Fauconneau et Pion.

En outre, ces expériences ont également pour but la détermination du niveau de l'azote endogène et de ses variations.

\section{MATÉRIEL ET MÉTHODES}

La méthode utilisée consiste à sacrifier les animaux à divers intervalles de temps après les repas expérimentaux ( 0 à 18 heures) et à prélever quantitativement et séparément les contenus digestifs des divers segments du tube digestif.

Cette technique a été utilisée sur vingt couples de porcs, l'aliment protidique étudié étant une farine de poisson.

Les animaux reçoivent pendant 8 jours avant leur abattage, un régime semi-purifié à $10 \%$ de protéine $(14 \%$ de farine de poisson, $3 \%$ de mélange minéral et vitaminique, $5 \%$ de cellulose et $78 \%$ d'amidon de maïs), à raison de $1 \mathrm{~kg}$ par repas (3 repas par jour). Leur avant-dernier repas, administré 18 heures en moyenne avant le dernier repas, est marqué à l'oxyde de chrome (10 $\mathrm{g}$ de $\mathrm{Cr}_{2} \mathrm{O}_{3}$ pour $1 \mathrm{~kg}$ de matière fraîche). Leur dernier repas est constitué par le régime de base pour l'un des animaux, par un régime analogue mais dépourvu de protéines pour l'autre. Ils sont ensuite abattus après un laps de temps choisi.

\section{RÉSULTATS}

Les résultats actuellement obtenus ne peuvent être donnés qu'à titre indicatif, en raison de la forte variabilité individuelle enregistrée.

$1^{\circ}$ Répartition de la matière sèche et de l'azote des contenus digestifs entre les divers segments $d u$ tube digestif (tableaux 1 et 2 )

La quantité totale de matière sèche trouvée dans le tube digestif (tableau 1) diminue quand le laps de temps écoulé depuis le repas s'allonge. Cette matière sèche est répartie dans les différents segments d'une manière qui se modifie avec le temps.

Malgré des variations individuelles importantes, on peut considérer que la quantité contenue dans le gros intestin est relativement constante dans les huit heures qui suivent 
le repas : d'après les résultats obtenus par ailleurs, indiquant que le coefficient d'utilisation digestive du régime est supérieur à 90 p. 100 , elle représente l'indigestible (300 à $400 \mathrm{~g}$ ) de 4 à 5 repas de $880 \mathrm{~g}$ (en matière sèche). En pourcentage de l'ensemble, son importance grandit au fur et à mesure que la quantité totale de matière sèche diminue dans le tube digestif.

La quantité de matière sèche trouvée dans l'intestin grêle représente un pourcentage relativement constant, mais faible de l'ensemble : les quantités sont de plus en plus petites lorsque augmente le temps écoulé depuis le repas.

Tableau 1

Répartition de la matière sèche des contenus entre les différents segments digestifs *.

\begin{tabular}{|c|c|c|c|c|c|c|c|c|c|}
\hline \multicolumn{2}{|c|}{ Temps après le repas $(h) \ldots$} & 1 & 2 & 3 & 4 & 5 & 6 & 7 & 8 \\
\hline \multicolumn{2}{|c|}{$\begin{array}{l}\text { 1) RÉGIME AZOTÉ : } \\
\text { Nombre d'animaux } \ldots \ldots \ldots\end{array}$} & 1 & 1 & 6 & 2 & 3 & 0 & 1 & 2 \\
\hline \multirow{2}{*}{ Estomac } & g & 716,0 & 496,6 & 452,1 & 366,7 & 431,3 & - & 159,9 & 76,2 \\
\hline & $\%$ du total & 59,3 & 50,6 & 47,2 & 47,5 & 51,0 & - & 28,6 & 14,2 \\
\hline \multirow{2}{*}{ Intestin grêle } & $\mathrm{g}$ & 184,2 & 119,8 & 143,5 & 138,5 & 88,6 & - & 82,9 & 76,5 \\
\hline & $\%$ du toral & 15,3 & 12,2 & 15,5 & 18,1 & 10,8 & - & 14,8 & 13,4 \\
\hline \multirow{2}{*}{ Gros intestin } & $\mathrm{g}$ & 306,7 & 365,0 & 355,7 & 265,2 & 315,4 & - & 316,4 & 400,2 \\
\hline & $\%$ du total & 25,4 & 37,2 & 37,3 & 34,4 & 38,2 & 一 & 56,6 & 72,4 \\
\hline \multicolumn{2}{|l|}{ Total. . } & 1206,9 & 981,4 & 951,3 & 770,4 & 835,3 & - & 559,2 & 552,9 \\
\hline \multicolumn{2}{|c|}{$\begin{array}{l}\text { 2) RÉGIME PROTÉIPRIVE : } \\
\text { Nombre d'animaux } \ldots \ldots \ldots\end{array}$} & 2 & 0 & 6 & 3 & 3 & 0 & 1 & 2 \\
\hline \multirow{2}{*}{ Estomac } & $\mathrm{g}$ & 605,9 & - & 453,3 & 336,4 & 286,5 & - & 265,5 & 253,2 \\
\hline & $\%$ du total & 52,9 & - & 49,4 & 49,7 & 38,0 & - & 34,5 & 38,8 \\
\hline \multirow{2}{*}{ Intestin grêle } & $\mathrm{g}$ & 268,2 & - & 140,3 & 94,2 & 79,3 & - & 64,3 & 65,4 \\
\hline & $\%$ du total & 23,3 & - & 16,2 & 13,8 & 11,1 & - & 8,3 & 11,6 \\
\hline \multirow{2}{*}{ Gros intestin } & $\mathrm{g}$ & 272,6 & - & 320,2 & 248,1 & 377,3 & - & 439,6 & 327,2 \\
\hline & $\%$ du total & 23,8 & - & 34,4 & 36,5 & 50,9 & - & 57,2 & 49,6 \\
\hline \multicolumn{2}{|c|}{ Total................. } & 1146,7 & - & 913,8 & 678,7 & 743,1 & 一 & 769,4 & 645,8 \\
\hline
\end{tabular}

* Ingéré $=880 \mathrm{~g}$ de matière sèche. 
Les quantités présentes au niveau de l'estomac représentent un pourcentage rapidement décroissant de l'ensemble des contenus digestifs; c'est à ce niveau que les variations en fonction du temps présentent la plus grande amplitude (de 10 à 1).

En ce qui concerne l'azote, les mêmes remarques générales peuvent être faites. Cependant, les quantités présentes dans l'intestin grêle représentent une fraction plus importante de l'ensemble que dans le cas de la matière sèche.

TABleau 2

Répartition de l'azote des contenus entre les différents segments digestifs.

\begin{tabular}{|c|c|c|c|c|c|c|c|c|c|}
\hline \multicolumn{2}{|c|}{ Temps après le repas $(h) \ldots$} & 1 & 2 & 3 & 4 & 5 & 6 & 7 & 8 \\
\hline \multicolumn{2}{|c|}{$\begin{array}{l}\text { 1) RÉGIME AZOTÉ : } \\
\text { (ingéré azoté : 17,3 g) }\end{array}$} & & & & & & & & \\
\hline & 1 & 1 & 6 & 2 & 3 & 0 & 1 & 2 \\
\hline \multirow{2}{*}{ Estomac } & $\mathrm{g}$ & 11,6 & 8,5 & 7,4 & 6,2 & 6,1 & - & 2,4 & 1,5 \\
\hline & $\%$ du total & 49,3 & 35,4 & 30,2 & 27,3 & 31,8 & - & 20,3 & 10,5 \\
\hline \multirow{2}{*}{ Intestin grêle } & $\mathrm{g}$ & 4,9 & 4,6 & 7,2 & 7,5 & 4,1 & - & 2,8 & 2,5 \\
\hline & $\%$ du total & 21,0 & 19,2 & 30,2 & 33,0 & 20,8 & - & 23,7 & 13,5 \\
\hline \multirow{2}{*}{ Gros intestin } & g & 7,0 & 10,9 & 9,9 & 9,0 & 8,9 & - & 6,6 & 11,5 \\
\hline & $\%$ du total & 29,7 & 45,4 & 39,6 & 39,7 & 47,4 & - & 56,0 & 76,0 \\
\hline \multicolumn{2}{|l|}{ Total. } & 23,5 & 24,0 & 24,5 & 22,7 & 19,1 & 一 & 11,8 & 15,5 \\
\hline \multicolumn{2}{|c|}{$\begin{array}{l}\text { 2) RÉGIME PROTÉlPRIVE : } \\
\text { Nombre d'animaux } \ldots \ldots \ldots\end{array}$} & 2 & 0 & 6 & 3 & 3 & 0 & 1 & 2 \\
\hline \multirow{2}{*}{ Estomac } & $\mathrm{g}$ & 1,9 & - & 1,4 & 1,1 & 1,0 & - & 0,9 & 1,7 \\
\hline & $\%$ du total & 13,8 & - & 13,7 & 10,5 & 7,8 & - & 6,4 & 25,5 \\
\hline \multirow{2}{*}{ Intestin grêle } & $\mathrm{g}$ & 5,1 & - & 4,7 & 4,7 & 3,4 & - & 2,9 & 2,1 \\
\hline & $\%$ du total & 33,6 & - & 30,9 & 38,2 & 25,3 & - & 19,9 & 27,9 \\
\hline \multirow{2}{*}{ Gros intestin } & g & 8,3 & - & 8,9 & 5,9 & 8,6 & - & 10,7 & 6,6 \\
\hline & $\%$ du total & 52,6 & - & 55,4 & 51,3 & 66,9 & - & 73,7 & 46,6 \\
\hline \multicolumn{2}{|c|}{ Total................... } & 15,3 & - & 15,0 & 11,7 & 13,0 & - & 14,5 & 10,4 \\
\hline
\end{tabular}




\section{$2^{\circ}$ Position des matières alimentaires provenant de l'avant-dernier repas}

Cette situation est précisée grâce au marqueur à l'oxyde de chrome. En moyenne, $86 \%$ du chrome ingéré lors de l'avant-dernier repas sont retrouvés dans le tube digestif, mais il existe d'importantes variations individuelles. S'il n'y a aucune relation entre la quantité retrouvée et le temps écoulé depuis ce repas (variant entre 15 et $22 \mathrm{~h}$ ), les variations individuelles peuvent être attribuées soit à un transit plus ou moins rapide et à un début d'excrétion, soit à la difficulté d'obtenir une récolte quantitative des contenus digestifs, particulièrement au niveau du gros intestin. C'est en effet dans cet organe (caecum, côlon, rectum) que se retrouve la plus grande partie du chrome $(96 \%$ en moyenne) présent dans l'ensemble du tube digestif. Le reste du chrome représente les traces trouvées dans les divers organes et correspond probablement à des particules retenues dans les replis de la muqueuse digestive.

Cet ensemble de faits signifie que, 15 à 22 heures après un repas, la presque totalité du reliquat d'un régime semi-purifié est parvenue au gros intestin; il est possible qu'une petite fraction en soit excrétée.

En ce qui concerne le principe de la méthode de marquage, il faut préciser que, chez deux animaux n'ayant pas reçu ultérieurement d'autres repas, un pourcentage important du chrome retrouvé quinze heures après le repas marqué, se trouve dans l'estomac (20\% environ). Ce fait semble prouver que le marqueur présente une vitesse de transit qui lui est propre et qu'il peut être amené à séjourner dans certains organes; le repas suivant chasserait, dans sa première vague, les particules résiduelles du traceur. Cette constatation demanderait à être vérifiée.

\section{$3^{\circ}$ Disparition de la matière sèche et de l'azote contenus dans l'estomac}

Les données concernant les quantités de matière sèche et d'azote retrouvées dans l'estomac ont été regroupées par unité de temps écoulé depuis le repas : elles se trouvent rapportées, en valeur absolue dans les tableaux 1 et 2 , en pourcentage de l'ingéré dans le tableau 3.

\section{Tableau 3}

Cinétique de la vidange stomacale (matière sèche et azote).

\begin{tabular}{|c|c|c|c|c|c|c|}
\hline \multirow{2}{*}{$\begin{array}{l}\text { Temps après } \\
\text { le repas }\end{array}$} & \multicolumn{2}{|c|}{ Nombre d'animaux } & \multicolumn{2}{|c|}{$\begin{array}{c}\text { Matière sèche dans l'estomac } \\
\% \text { de l'ingéré* }\end{array}$} & \multirow{2}{*}{$\begin{array}{c}\text { Azote dans } \\
\text { l'estomac } \\
\% \\
\text { de l'ingéré** } \\
\text { (régime azoté) }\end{array}$} & \multirow{2}{*}{$\begin{array}{l}\text { Pourcentage } \\
\text { estimé d'azote } \\
\text { endogène } \\
\text { (régime azoté) }\end{array}$} \\
\hline & $\begin{array}{l}\text { Régime } \\
\text { azoté }\end{array}$ & $\begin{array}{c}\text { Régime } \\
\text { protéiprive }\end{array}$ & $\begin{array}{l}\text { Régime } \\
\text { azoté }\end{array}$ & $\begin{array}{c}\text { Régime } \\
\text { protéiprive }\end{array}$ & & \\
\hline $1 \mathrm{~h}$ & 1 & 2 & 81,4 & 68,8 & 67,0 & 16 \\
\hline $2 \mathrm{~h}$ & 1 & 一 & 56,4 & 一 & 49,1 & - \\
\hline $3 \mathrm{~h}$ & 6 & 6 & 51,4 & 51,5 & 42,9 & 19 \\
\hline $4 \mathrm{~h}$ & 2 & 3 & 41,7 & 38,2 & 35,8 & 18 \\
\hline $5 \mathrm{~h}$ & 3 & 3 & 49,0 & 32,5 & 35,1 & 16 \\
\hline $6 \mathrm{~h}$ & 一 & - & - & - & 一 & 一 \\
\hline $7 \mathrm{~h}$ & 1 & 1 & 18,2 & 30,2 & 14,0 & 38 \\
\hline $8 \mathrm{~h}$ & 2 & 2 & 8,6 & 28,8 & 8,5 & $>100$ \\
\hline
\end{tabular}

* Ingéré $=880 \mathrm{~g}$.

* Ingéré $=17,3 \mathrm{~g}$. 


\section{Matière sèche}

Une fraction importante de la matière sèche ingérée (45\%) disparaît de l'estomac dans les deux heures qui suivent le repas contenant la protéine; puis survient une phase apparemment stationnaire ( 2 à $5 \mathrm{~h}$ après le repas) au cours de laquelle ne se produit qu'une faible vidange; enfin, il se produit à nouveau une disparition rapide de matière sèche : 8 heures après le repas ne subsistent plus dans l'estomac que $10 \%$ environ de la matière sèche ingérée. Le phénomène présente la même allure générale pour le régime protéiprive; cependant, la vidange paraît initialement plus rapide, mais se ralentit progressivement pour devenir nulle 5 heures après le repas; 8 heures après le repas se retrouvent encore dans l'estomac $30 \%$ du repas protéiprive ingéré.

Azote

Chez les animaux recevant le régime azoté, la vidange d'azote, plus rapide que celle de matière sèche, est aussi un phénomène plus continu; elle présente néanmoins le même aspect général que la vidange de matière sèche.

En régime protéiprive, la quantité d'azote présente dans l'estomac n'est pas négligeable; elle diminue en valeur absolue dans les 7 heures qui suivent le repas. Si l'on admet l'hypothèse selon laquelle le niveau de l'azote endogène (secrétions et desquamations) est le même en régimes azoté et protéiprive, la fraction d'azote endogène représente 15 à $20 \%$ de l'azote trouvé dans les contenus stomacaux dans les 5 heures suivant un repas azoté. Cette fraction s'élève progressivement entre 5 heures et 8 heures après le repas pour représenter en fin de compte la totalité de l'azote stomacal.

On peut élever certaines critiques sur la présentation des résultats sous forme de moyennes qui tient trop compte des variations individuelles étant donné le nombre de données pour chacun des temps après le repas. C'est pourquoi, bien que la vidange stomacale que l'on peut décrire d'après ces résultats moyens soit un phénomène apparemment discontinu, nous avons cherché à savoir s'il existait une relation mathématique

\section{TABleau 4}

Relations entre les quantités d'azote et de matière sèche présentes dans divers segments du tube digestif et le temps écoulé depuis le dernier refas.

\begin{tabular}{|c|c|c|}
\hline Organe & Régime azoté & Régime protéiprive \\
\hline Estomac & $\begin{aligned} 1(n=18) \log \mathrm{N} & =4,16-10^{-3} \times 1,80 t \\
r & =-0,84 * * \\
2(n=18) \log \text { M.S. } & =2,94-10^{-3} \times 1,76 t \\
r & =-0,76^{* *}\end{aligned}$ & $\begin{aligned} 5(n=20) \log \mathrm{N} & =3,07-10^{-3} \times 0,04 t \\
r & =-0,03 \text { N.S. } \\
6(n=20) \log \text { M.S. } & =2,62-10^{-3} \times 0,57 t \\
r & =-0,27 \text { N.S. }\end{aligned}$ \\
\hline Intestin grêle & $\begin{aligned} 3(n=17) \log \mathrm{N} & =3,99-10^{-3} \times 1,09 t \\
r & =-0,65^{* *} \\
4(n=18) \log \text { M.S. } & =2,32-10^{-3} \times 1,13 t \\
r & =-0,60^{* *}\end{aligned}$ & $\begin{array}{c}7(n=17) \log \mathrm{N}=3,73-10^{-3} \times 0,78 t \\
r=-0,38 \mathrm{~N} . \mathrm{S} . \\
8(n=20) \log \text { M.S. } \\
=2,18-10^{-3} \times 0,85 t \\
r=--0,48^{*}\end{array}$ \\
\hline
\end{tabular}

* Seuil de signification 0,05 .

*** " $" 0,01$. 
entre les quantités trouvées dans l'estomac (exprimées en logarithmes) et le laps de temps écoulé depuis le repas. De fait, il existe une régression entre les deux variables qui est traduite par les équations rapportées dans le tableau 4 et illustrée dans les graphiques 1 et 2 . Il est à noter que le coefficient de corrélation est élevé et très significatif pour les animaux recevant les régimes azotés, ce qui n'est pas le cas pour les animaux recevant les régimes protéiprives.

On peut donc penser que, pour les premiers, la vidange stomacale peut être traduite par une courbe du type exponentiel, ce qui confirme les résultats de HunT et SPURRELL (1951) et tendrait à prouver que c'est un phénomène continu. Pour les seconds, il est possible que le fait de ne pas les avoir accoutumés au régime protéiprive provoque des perturbations du transit de la matière sèche; par ailleurs, les quantités d'azote endogène trouvées dans l'estomac seraient relativement constantes dans le temps.

\section{$4^{\circ}$ Azote et matière sèche dans l'intestin grêle}

Les quantités de matière sèche trouvées dans l'intestin grêle (tableau 1) sont beaucoup plus faibles que celles trouvées dans l'estomac au cours des six heures qui suivent l'ingestion du repas. En outre, l'amplitude des variations au cours du temps est réduite.

En ce qui concerne l'azote, les quantités trouvées dans l'intestin grêle (tableau 2) sont toujours importantes, en particulier dans sa première partie (duodenum); ce fait implique une concentration de l'azote au sein de la matière sèche. On peut penser que ce phénomène est lié au niveau de l'azote endogène (en particulier des sécrétions digestives) : si l'on se réfère à ce qui se passe dans le tube digestif des animaux recevant le régime protéiprive, 60 à $100 \%$ de l'azote trouvé dans l'intestin grêle des animaux recevant le régime azoté (tableau 5) pourraient provenir de l'organisme.

\section{Tableau 5}

Pourcentage estimé d'azote endogène dans les contenus d'intestin grêle (régime azoté).

\begin{tabular}{|c|c|c|c|c|c|c|c|c|}
\hline Temps après le repas (h) & 1 & 2 & 3 & 4 & 5 & 6 & 7 & 8 \\
\hline $\begin{array}{l}\text { Pourcentage : } \\
\mathrm{N} \text { régime protéiprive } \\
\mathrm{N} \text { régime azoté }\end{array}$ & $>100$ & 一 & 66 & 62 & 82 & 一 & $>100$ & 85 \\
\hline
\end{tabular}

Dans le tableau 4 sont rapportées les équations de régression entre le logarithme des quantités d'azote et de matière sèche trouvées dans l'intestin grêle et le temps écoulé depuis le repas (illustrations dans les graphiques 3 et 4).

Ces formules montrent que les quantités de matière sèche et d'azote trouvées dans l'intestin grêle diminuent lorsque s'allonge le laps de temps écoulé depuis le repas. Le phénomène étant plus rapide en régime azoté qu'en régime protéiprive, il faut préciser que, dans ce dernier cas, le coefficient de corrélation $r$ n'est pas significatif pour le cas de la matière sèche, ce qui était déjà trouvé pour les équations traduisant la vidange stomacale dans les régimes protéiprives. 
En résumé, l'étude du transit digestif à l'aide de la technique d'abattages échelonnés dans le temps après le repas s'est révélée longue et laborieuse et il serait bon d'y substituer une technique plus élaborée comme, par exemple, la fistulation digestive en divers points.

Les faits acquis dans cette étude sont les suivants :

- La vidange stomacale semble un phénomène continu, dont l'évolution dans le temps suit une courbe du type exponentiel. Le niveau de l'azote d'origine endogène est relativement important au niveau de l'estomac.

- La matière sèche trouvée au niveau de l'intestin grêle est peu abondante et très concentrée en azote (dont la majeure partie semble être d'origine endogène). Les quantités totales trouvées à ce niveau diminuent quand s'allonge le laps de temps écoulé après le repas.

- La quantité de matière sèche trouvée dans le gros intestin est relativement constante. C'est à ce niveau que se trouve, dans sa quasi totalité, l'avant-dernier repas ingéré (15 à $22 \mathrm{~h}$ auparavant). 


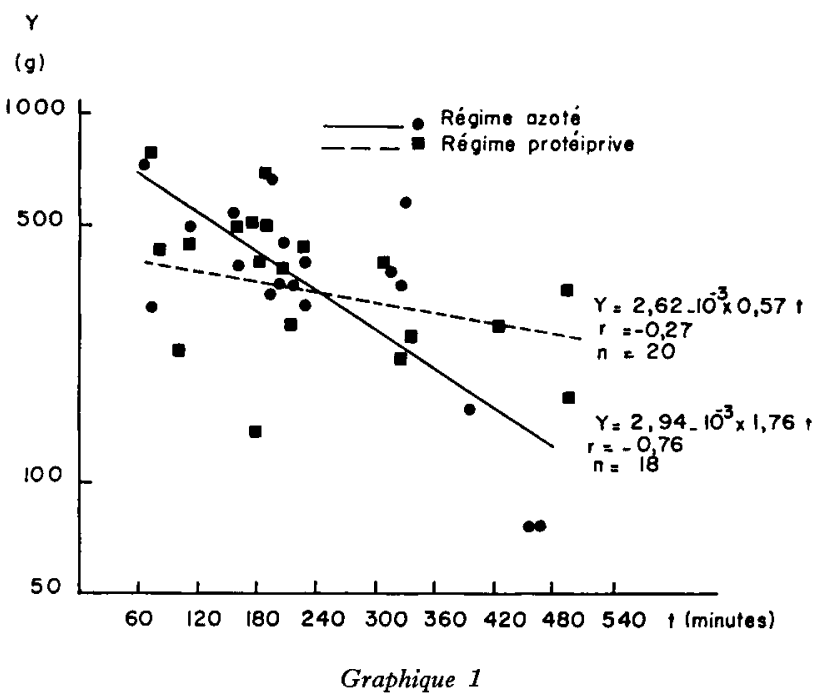

Quantité de matière sèche dans l'estomac.

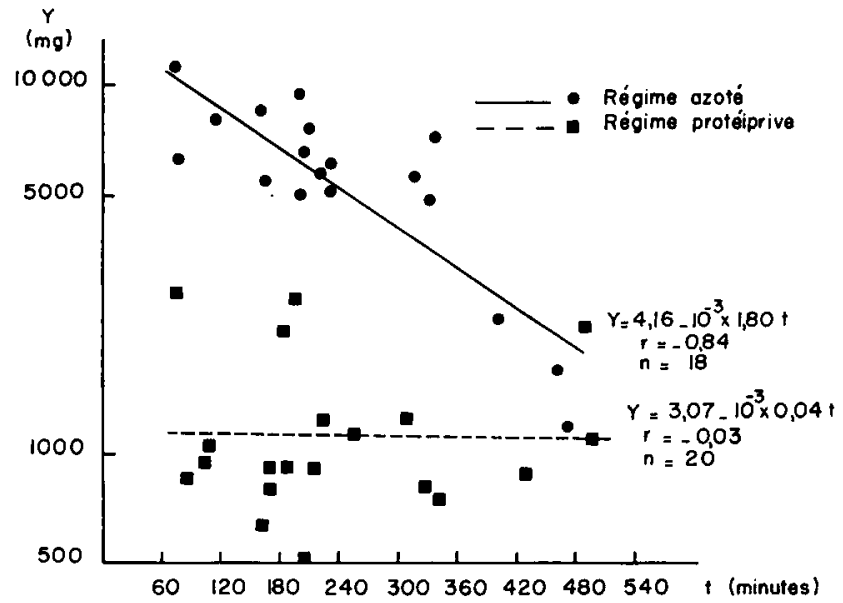

Graphique 2

Quantité d'azote dans l'estomac. 


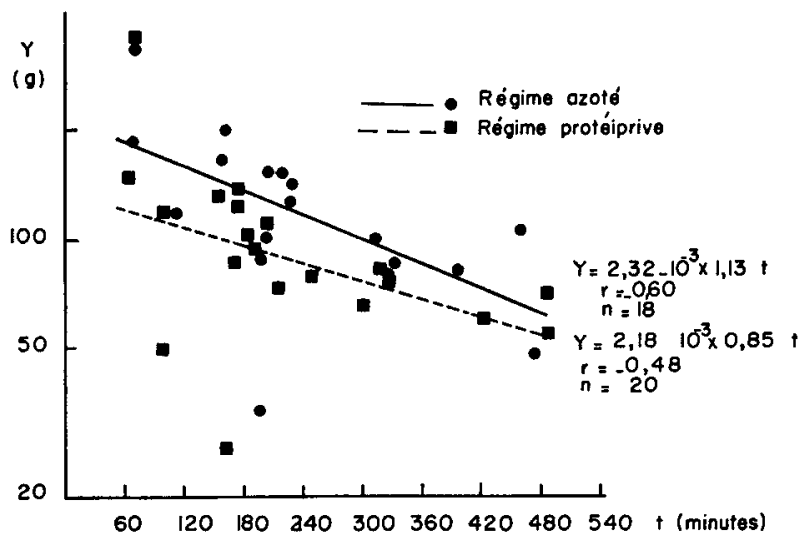

Graphique 3

Quantité de matière sèche dans l'intestin grêle.

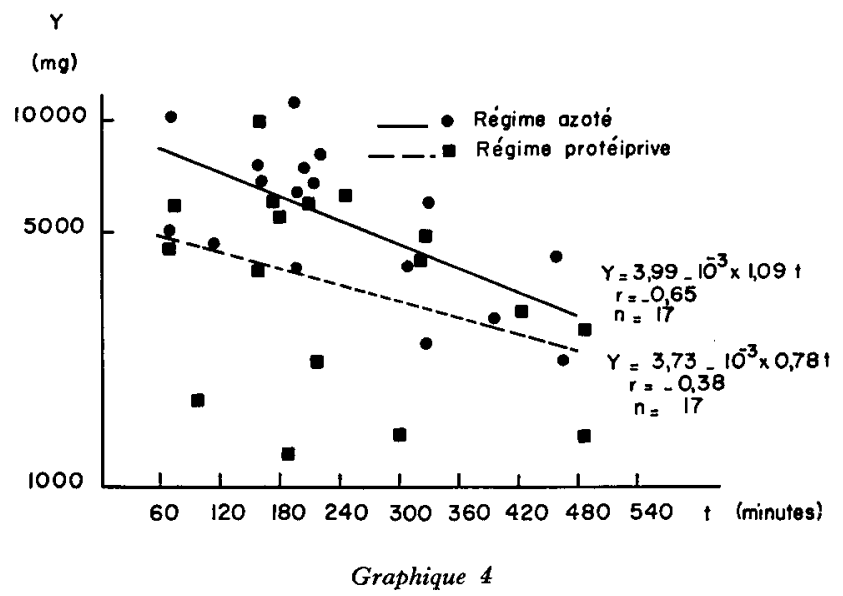

Quantité d'azote dans l'intestin grêle. 